\title{
Low-to-moderate alcohol intake and breast cancer risk in Chinese women
}

\author{
M Zhang', and CDJ Holman' \\ 'School of Population Health, The University of Western Australia, 35 Stirling Highway, Crawley, Perth, Western Australia 6009, Australia
}

\begin{abstract}
BACKGROUND: Despite extensive investigation of the association between alcohol consumption and breast cancer risk, effect of low-to-moderate alcohol intake on breast cancer incidence has been inconsistent.

METHODS: A case-control study was conducted in China, 2004-2005 to examine the association by menopausal status, oestrogen (ER) and progesterone receptor (PR) status of the tumour. There were 1009 incident cases with histologically confirmed breast cancer and 1009 age-matched controls recruited. We assessed alcohol consumption by face-to-face interview using a validated questionnaire and obtained tumour ER and PR status from pathology reports.

RESULTS: Low-to-moderate alcohol consumption was inversely associated with breast cancer risk. Compared with nondrinkers, the adjusted odds ratios (ORs) for alcohol $<5$ g per day were 0.41 (95\% confidence interval $0.27-0.62$ ) and 0.62 (0.48-0.79) in postmenopausal and premenopausal women, respectively. The inverse association was consistent for alcohol $<15 \mathrm{~g}$ per day across hormone receptor status groups with ORs of $0.36-0.56$ in postmenopausal women and 0.57-0.64 in premenopausal women. An exception was that alcohol $\geqslant 15 \mathrm{~g}$ per day appeared to increase the risk of breast cancers with discordant receptor status in postmenopausal women, that is, ER+/PR- or ER-/PR + (4.27, 1.57-1 I.65).

CONCLUSION: We found that low-to-moderate alcohol intake was not associated with increased risk of breast cancer in pre- or postmenopausal Chinese women. Future studies are required to understand differences in effect of alcohol on breast cancers by tumour hormone receptor status.

British Journal of Cancer (201I) 105, 1089-1095. doi:10.1038/bjc.2011.302 www.bjcancer.com
\end{abstract}

Published online 9 August 201 I

(c) 201। Cancer Research UK

Keywords: alcohol consumption; breast cancer; Chinese women; hormone receptor

Despite extensive research on the association between alcohol consumption and breast cancer risk, a consensus is not apparent, especially on the effect of low-to-moderate alcohol intake (Brown et al, 2010). Since a meta-analysis provided evidence for a doseresponse relation, admittedly with a weak ascending slope, alcohol has been treated as a risk factor for breast cancer even at low-tomoderate daily intake levels (Longnecker, 1994). Alcohol consumption has been reported to be associated with higher risk of breast cancer incidence (Smith-Warner et al, 1998; Bagnardi et al, 2001; Terry et al, 2006). However, subsequent studies based on more detailed data on consumption habits have not consistently supported the finding, particularly in premenopausal women (Garland et al, 1999; Kinney et al, 2000; Kropp et al, 2001; Baumgartner et al, 2002; Nagata et al, 2007; Bessaoud and Daurès, 2008; Brown et al, 2010; Kabat et al, 2010). Thus, the effect of low-to-moderate alcohol intake on breast cancer risk remains contentious.

Little is known about the relationship between alcohol intake and breast cancer risk in Chinese women, a population that consumes alcohol at very low levels (WHO, 2004). WHO reported that the lifetime prevalence of abstainers was $61.2 \%$ and that only $2.1 \%$ of Chinese women consumed three or more standard drinks

*Correspondence: Dr M Zhang; E-mail: min.zhang@uwa.edu.au Received 22 February 201 I; revised 23 June 2011; accepted 30 June 201 I; published online 9 August 201 I on a typical drinking day (WHO, 2004). There have been a few studies on alcohol consumption and breast cancer risk in Chinese women. One study in premenopausal Vietnamese and Chinese women reported that those who consumed alcohol had an increased risk of breast cancer compared with women who did not (Nichols et al, 2005). However, a recent study suggested that low alcohol intake was not related to increased breast cancer risk in Asian Americans including Chinese women (Brown et al, 2010).

Considering that alcohol consumption is one of the few modifiable factors associated with breast cancer risk, investigations to further clarify this issue are warranted. We conducted a case-control study in southeast China specifically to examine associations between alcohol intake, primarily low-to-moderate levels and breast cancer risk in the population using a validated questionnaire. The analyses were stratified according to pre- and postmenopausal status and the study also explored whether differences exist in the effect of alcohol according to oestrogen (ER) and progesterone receptor (PR) status of the tumour.

\section{PARTICIPANTS AND METHODS}

\section{Study design and participants}

A hospital-based case-control study of breast cancer risk was conducted in Hangzhou, the capital city of Zhejiang Province, during July 2004 and September 2005. All participants were 
Chinese women resident in Zhejiang Province and aged between 20 and 87 years. Cases were identified from medical records in four teaching hospitals of the School of Medicine, Zhejiang University. All the participating hospitals were public hospitals with 500-2000 beds and received patients from all over the province. A total of 1009 female patients, who newly diagnosed with invasive ductal carcinomas or in situ carcinoma of the breast, were recruited to the study. All diagnoses were histopathologically confirmed after surgery. The diagnoses occurred no more than 1 year before the interview and there was no previous diagnosis of cancer at any site. The patients were excluded if breast cancer was neither the primary nor final diagnosis. All relevant hospital and laboratory pathology reports were reviewed daily to ensure the completion of recruitment. The proportion of lost or non-responding patients among the cases was $1.2 \%$. Among 1009 cases recruited, we obtained information on ER status in 756 cases $(74.9 \%)$ and PR status in 755 cases $(74.8 \%)$ from pathology reports filed in the patients' medical records of immunohistochemistry assays undertaken at the time of diagnosis. Oestrogen receptor levels obtained from the pathology reports referred to the $\alpha$-subtype of the receptor.

During the same period of data collection, 1009 outpatients who remained healthy were selected consecutively in the participating hospitals as controls to match each case's age within 5-year age groups, using a daily update of the list of cases after they had consulted their doctors. Each control was recruited as the first in the matched age group to attend the breast clinic for routine preventive care and who consented to participate. The proportion of selected controls who participated was $98.7 \%$. Potential control women were excluded if they had a diagnosis of any benign or neoplastic breast disease, or another malignant disease at the time of recruitment. The project received ethics clearance from both the Human Research Ethics Committee of The University of Western Australia and the Chinese hospital authorities.

\section{Questionnaire and interview}

Subjects were briefed regarding the general aims of the study to investigate lifestyle factors. An appointment for an interview was made after obtaining their consent at initial contact. A face-to-face interview was then conducted by trained staff using a structured questionnaire that usually took $40-50 \mathrm{~min}$. The cases were interviewed in breast surgery wards and most of them (91.6\%) within 3 months after diagnosis, while the controls were interviewed in the outpatient clinic of the same hospital. A validated and reliable questionnaire was used to collect the information on: (a) demographic and lifestyle characteristics, for example, residential area, education, weight and height, smoking, alcohol consumption, tea drinking, and physical activity; (b) habitual dietary intake assessed by a 100 -item food frequency questionnaire (FFQ); and (c) factors relevant to hormonal status, including menstrual history and menopausal status, reproductive and lactation history, hormone replacement treatment, history of use of oral contraceptives, other factors relevant to hormonal status, benign proliferative breast disease and family history of breast cancer. After interview, anthropometric measurements were requested of all participants.
Alcohol consumption was assessed using the FFQ to measure three different types of alcoholic beverages, that is, beer, wine and liquor. The frequency of consumption was assigned to one of nine categories of never or hardly ever, once a month, 2-3 times a month, once a week, 2-3 times a week, 4-6 times a week, once a day, twice a day and $\geqslant 3$ times a day. Information was also sought on quantities consumed of alcohol in ml. Standard drinking vessels used by Zhejiang residents were displayed during the interview to increase the accuracy of measurement. Alcohol consumption was based on a usual drinking pattern and a 'reference' recall period was set as 1 year before diagnosis in cases or interview in controls. If there was any recent change in habits or quitting drinking, information was sought on the respondent's habits before the change and the year of quitting.

The questionnaire was adapted from that used in our previous studies on cancers (Zhang et al, 2002). The questionnaire was first pre-tested on 51 adult Chinese women who recently migrated to Perth, Australia, to assess the feasibility, face and content validity. The participants in the preliminary study reported their alcohol consumption when they were in China. The feedback from the participants indicated that they could estimate frequency and quantity of beer, wine and liquor consumed without difficulty. A test-retest study was then undertaken to assess the reliability of the questionnaire. Another sample of 41 Chinese women residing in Hangzhou was interviewed twice within 11.3 (s.d. 6.2) weeks (Zhang et al, 2005). No significant difference was found in alcohol consumption variables between the two interviews. Intraclass correlations ranged from 0.51 for wine intake to 0.87 for beer intake. The results, shown in Table 1, thus suggested that the questionnaire was a suitable and reproducible instrument to measure alcohol exposure for Chinese women.

\section{Statistical analysis}

All data were checked for completeness at the end of each interview. The data were coded and analysed using the SPSS version 17.0 (SPSS Inc., New York, NY, USA). Data collected by different interviewers were compared and we confirmed that no consistent difference in recording key variables, such as alcohol consumption, tea drinking and physical activity, occurred within cases or controls. The frequency and quantity variables derived from the FFQ were converted into daily intake (in $\mathrm{ml}$ ) of beer, wine and liquor. Amounts of ethanol intake were calculated by assuming $10 \mathrm{~g}$ of ethanol per $285 \mathrm{ml}$ of beer, per $100 \mathrm{ml}$ of wine and per $30 \mathrm{ml}$ of liquor based on a method used in a previous study (Kropp et al, 2001). Food consumption was adjusted for the edible portions of foods, cooking methods, seasonal factors and market availability (Whitemore et al, 1990). Total energy intake was estimated using Chinese Food Composition Tables (Institute of Nutrition and Food Hygiene, 1999). Intakes of folate in $\mu \mathrm{g}$ were calculated based on daily food consumption, using an updated version of the USDA nutrient database (USDA, 2007). The mean daily intakes of folate were tabulated separately for case and control groups. The quantitative variables of folate intake were divided into tertiles based on the corresponding empirical distribution in controls, with the low tertile being the reference category. Some category variables were defined as: a total of 20

Table I Reliability assessment for alcohol intake in the test-retest study

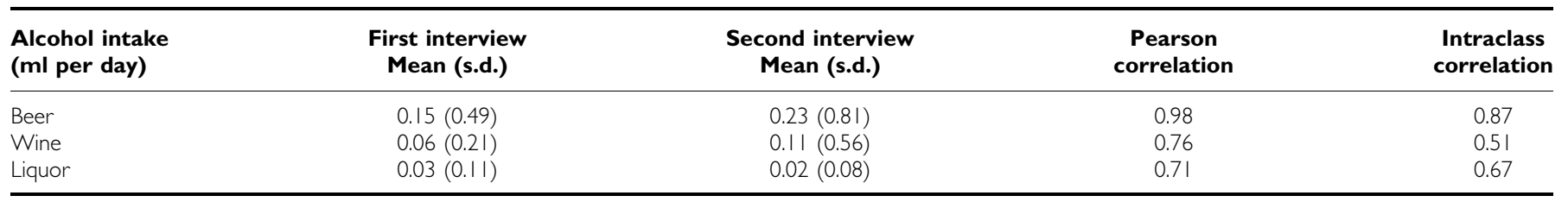


packs of cigarettes or more in a lifetime for tobacco smoking; and BMI calculated from reported body height and weight using the formula for Quetelet's index (expressed in $\mathrm{kg} \mathrm{m}^{-2}$ ). Physical activity was expressed in weekly metabolic equivalent task (MET) hours (Zhang et al, 2003). Metabolic equivalent task scores 6, 4.5 and 2.5 were assigned respectively for vigorous, moderate and walking activity based on a compendium of physical activities (Ainsworth et al, 2000). Patients were defined as postmenopausal if they indicated that they had ceased having periods for at least 1 year before the interview. Those patients who had had a surgical menopause at age over 50 years were also assigned to the postmenopausal group.

Distributions of demographic characteristics and potential risk factors of cases and controls were compared using the $t$-test for continuous variables and $\chi^{2}$-test for categorical variables. Odds ratios (ORs) and 95\% confidence intervals (CIs) for each level of alcohol intake were estimated using unconditional logistic regression including the matching factors as covariates. Univariate analysis was undertaken to screen potential explanatory variables for subsequent multivariate analysis. In each analysis, effects of alcohol of different patterns and intake levels were assessed within current drinkers, excluding ex-drinkers (1.2\% of participants), with abstainers who never drank alcohol as a reference group. We examined potential confounders of the alcohol effect through use of stratified analysis by menopausal status (premenopausal vs postmenopausal). Separate sub-analyses were conducted for each subtype of tumour hormone status (ER and PR separately and jointly). Each fitted regression equation was adjusted for age, education, BMI, oral contraceptive use, hormone replacement therapy, breast cancer in first-degree relatives, total energy intake, folate intake, tea drinking and menopausal status (only included the models for all women). These potential confounders were included in the models, because either they emerged as risk factors for breast cancer in previous studies (Kropp et al, 2001) or because we observed some evidence of potential confounding in our data set by comparisons of univariate and multivariate analyses (Zhang et al, 2007, 2009). The proportion of in situ carcinoma was only $2.2 \%$ of all breast cancer patients in the study, making it impracticable to study them separately. Therefore, the patients with in situ carcinomas and invasive breast cancer were combined to form a single case group in analyses.

\section{RESULTS}

Selected demographic characteristics and lifestyle factors in cases with breast cancer and controls are compared in Table 2. Fewer breast cancer cases $(33.1 \%)$ were current drinkers compared with controls $(46.6 \%)$. Among current drinkers, the majority of participants $(77 \%$ cases, $87 \%$ controls) consumed $<10 \mathrm{~g}$ daily, in contrast to 39 cases $(12 \%)$ and 16 controls $(3 \%)$ who consumed $\geqslant 30 \mathrm{~g}$ daily. Cases had less education and tea drinking, and more use of oral contraceptives. More of cases had a higher BMI at 5 -years ago, breast cancer in a first-degree relative and energy intake. There was no difference between cases and controls in age at interview, locality, age at first full-term pregnancy, number of children breastfed, menopausal status, hormone replacement therapy, smoking, mean dietary folate intake and physical activity in terms of weekly MET-hours.

Table 3 reports associations between breast cancer risk and alcohol consumption. Compared with abstainers, the adjusted ORs in current drinkers were 0.66 (95\% CI $0.53-0.84)$ and $0.55(0.38-$ 0.78 ) for premenopausal and postmenopausal women. A significant inverse association was observed in premenopausal women who drank wine only $(0.30,0.17-0.52)$, and postmenopausal women who consumed beer only $(0.34,0.15-0.75)$. Compared with nondrinkers, those who consumed alcohol $<5 \mathrm{~g}$ per day had a reduced risk of breast cancer. The adjusted ORs (95\% CIs) were
Table 2 Selected characteristics of subjects with and without breast cancer

\begin{tabular}{|c|c|c|c|}
\hline & $\begin{array}{c}\text { Cases } \\
\text { subjects } \\
(n=1009)\end{array}$ & $\begin{array}{l}\text { Controls } \\
\text { subjects } \\
(n=1009)\end{array}$ & $P$-value ${ }^{a}$ \\
\hline $\begin{array}{l}\text { Age at interview (years) } \\
\text { Resident in rural area }\end{array}$ & $\begin{array}{l}48.4 \pm 10.3 \\
47 \mid(46.7)\end{array}$ & $\begin{array}{l}48.4 \pm 10.3 \\
475(47.1)\end{array}$ & $\begin{array}{l}0.96 \\
0.86\end{array}$ \\
\hline $\begin{array}{l}\text { Education } \\
\text { No formal education } \\
\text { Primary } \\
\text { Secondary } \\
\text { Tertiary }\end{array}$ & $\begin{array}{l}183(18.1) \\
290(28.7) \\
395(39.2) \\
141(14.0)\end{array}$ & $\begin{array}{l}151(15.0) \\
289(28.6) \\
376(37.3) \\
193(19.1)\end{array}$ & $<0.01$ \\
\hline $\begin{array}{l}\text { Body mass index } \\
\left(\mathrm{kg} \mathrm{m}^{-2}, 5 \text {-years ago }\right) \\
\quad<25 \\
\quad \geqslant 25\end{array}$ & $\begin{array}{l}794(78.7) \\
215(21.3)\end{array}$ & $\begin{array}{l}84 \mid(83.3) \\
168(16.7)\end{array}$ & $<0.01$ \\
\hline $\begin{array}{l}\text { No. of delivery of full-term } \\
\text { pregnancy }\end{array}$ & $1.70 \pm 1.2$ & $1.71 \pm 1.2$ & 0.94 \\
\hline $\begin{array}{l}\text { No. of children breastfed } \\
\text { Oral contraceptive use (year) } \\
\text { Hormone replacement } \\
\text { therapy (year) }\end{array}$ & $\begin{array}{l}1.61 \pm 1.1 \\
3.1 \pm 5.1 \\
1.7 \pm 3.4\end{array}$ & $\begin{array}{l}1.67 \pm 1.2 \\
2.3 \pm 3.6 \\
1.4 \pm 1.6\end{array}$ & $\begin{array}{l}0.24 \\
0.02 \\
0.67\end{array}$ \\
\hline $\begin{array}{l}\text { Postmenopausal status } \\
\text { Breast cancer in first-degree } \\
\text { relatives }\end{array}$ & $\begin{array}{r}337(33.4) \\
35(3.5)\end{array}$ & $\begin{array}{r}338(33.5) \\
17(1.7)\end{array}$ & $\begin{array}{l}0.96 \\
0.01\end{array}$ \\
\hline $\begin{array}{l}\text { Alcohol consumption } \\
\text { Abstainers } \\
\text { Ex-drinkers } \\
\text { Current drinkers }\end{array}$ & $\begin{array}{r}660(65.4) \\
15(1.5) \\
334(33.1)\end{array}$ & $\begin{array}{r}529(52.4) \\
10(1.0) \\
470(46.6)\end{array}$ & $<0.001$ \\
\hline 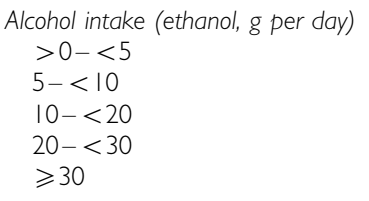 & $\begin{array}{r}225(67.4) \\
28(8.4) \\
12(3.6) \\
45(13.5) \\
39(11.7)\end{array}$ & $\begin{array}{r}366(77.9) \\
42(8.9) \\
13(2.8) \\
43(9.1) \\
16(3.4)\end{array}$ & $<0.001$ \\
\hline $\begin{array}{l}\text { Tea drinking } \\
\text { Tobacco smoking } \\
\text { Physical activity } \\
\text { (weekly MET-hour) }\end{array}$ & $\begin{array}{r}451(44.7) \\
20(2.0) \\
58.3 \pm 71.2\end{array}$ & $\begin{array}{r}661(65.5) \\
14(1.4) \\
60.1 \pm 59.1\end{array}$ & $\begin{array}{c}<0.001 \\
0.30 \\
0.54\end{array}$ \\
\hline $\begin{array}{l}\text { Folate intake ( } \mu \mathrm{g} \text { per day) } \\
\text { Energy intake (kcal per day) }\end{array}$ & $\begin{array}{c}577.5 \pm 168.5 \\
2184.0 \pm 569.3\end{array}$ & $\begin{array}{c}579.2 \pm 164.7 \\
2084.3 \pm 506.3\end{array}$ & $\begin{array}{c}0.82 \\
<0.001\end{array}$ \\
\hline
\end{tabular}

Abbreviation: $M E T=$ metabolic equivalent task. ${ }^{a}$ Two-sided, $t$-test for continuous variables and $\chi^{2}$ test for categorical variables. Values expressed as mean \pm s.d. or number (percent)

$0.41(0.27-0.62)$ and $0.62(0.48-0.79)$ in postmenopausal and premenopausal women, respectively. There was a marginal significant increased risk $2.28(1.00-5.20)$ for daily alcohol intake $\geqslant 30 \mathrm{~g}$ in premenopausal women.

Table 4 shows the combined effect of dietary folate intake and alcohol consumption on breast cancer risk. Folate intake was associated with a decreased risk of breast cancer in women who were abstainers or consumed alcohol $>0-<15$ g daily. Among the women who consumed alcohol $\geqslant 15 \mathrm{~g}$ daily, a decreased breast cancer risk was only observed in those who consumed high not low or middle tertile of folate intake $(\mathrm{OR}=0.38,95 \%$ CI $0.20-0.72$ ) and not in the low or middle tertiles.

Table 5 shows associations between alcohol intake and breast cancer within hormone receptor subtypes. Compared with nondrinkers, the effects of alcohol intake of $<15 \mathrm{~g}$ daily were protective in all subtypes defined by ER and PR status. For daily alcohol intake of $\geqslant 15 \mathrm{~g}$, an increased but nonsignificant association was found for all tumour receptor subtypes.

Table 6 reports adjusted ORs and 95\% CIs for alcohol intake and breast cancer risk by joint $\mathrm{ER}$ and $\mathrm{PR}$ status. Relative to nondrinkers, an inverse effect was associated with alcohol intake 
Table 3 Association between alcohol consumption and breast cancer risk

\begin{tabular}{|c|c|c|c|}
\hline & $\begin{array}{c}\text { No. } \\
\text { cases/controls }\end{array}$ & $\begin{array}{c}\text { OR } \\
(95 \% \mathrm{Cl})^{a}\end{array}$ & $P$-value \\
\hline \multicolumn{4}{|l|}{ Alcohol consumption } \\
\hline \multicolumn{4}{|l|}{ All women } \\
\hline Abstainers & $660 / 529$ & $1.00^{\mathrm{b}}$ & \\
\hline Ex-drinkers & $15 / 10$ & $1.34(0.56-3.22)$ & 0.51 \\
\hline Current drinkers & $334 / 470$ & $0.63(0.52-0.76)$ & $<0.001$ \\
\hline \multicolumn{4}{|c|}{ Premenopausal women } \\
\hline Abstainers & $416 / 332$ & $1.00^{\mathrm{b}}$ & \\
\hline Ex-drinkers & $10 / 4$ & $2.44(0.7 I-8.39)$ & 0.16 \\
\hline Current drinkers & $246 / 335$ & $0.66(0.53-0.84)$ & 0.001 \\
\hline \multicolumn{4}{|c|}{ Postmenopausal women } \\
\hline Abstainers & $244 / 197$ & $1.00^{\mathrm{b}}$ & \\
\hline Ex-drinkers & $5 / 6$ & $0.68(0.17-2.67)$ & 0.58 \\
\hline Current drinkers & $88 / 135$ & $0.55(0.38-0.78)$ & 0.001 \\
\hline \multicolumn{4}{|l|}{ Type of alcohol consumed } \\
\hline \multicolumn{4}{|l|}{ All women } \\
\hline Abstainers & $660 / 529$ & $1.00^{\mathrm{b}}$ & \\
\hline Beer only & $82 / 103$ & $0.65(0.47-0.90)$ & 0.01 \\
\hline Wine only & $37 / 91$ & $0.40(0.26-0.60)$ & $<0.001$ \\
\hline Liquor only & $30 / 30$ & $0.81(0.47-1.39)$ & 0.44 \\
\hline \multicolumn{4}{|c|}{ Premenopausal women } \\
\hline Abstainers & $416 / 332$ & $1.00^{\mathrm{b}}$ & \\
\hline Beer only & $70 / 81$ & $0.73(0.51-1.06)$ & 0.10 \\
\hline Wine only & $19 / 60$ & $0.30(0.17-0.52)$ & $<0.001$ \\
\hline Liquor only & $19 / 18$ & $0.73(0.37-1.47)$ & 0.38 \\
\hline \multicolumn{4}{|c|}{ Postmenopausal women } \\
\hline Abstainers & $244 / 197$ & $1.00^{\mathrm{b}}$ & \\
\hline Beer only & $12 / 22$ & $0.34(0.15-0.75)$ & $<0.01$ \\
\hline Wine only & $|8 / 3|$ & $0.61(0.32-1.16)$ & 0.13 \\
\hline Liquor only & $11 / 12$ & $0.88(0.36-2.16)$ & 0.77 \\
\hline \multicolumn{4}{|c|}{ Ethanol intake (g per day) } \\
\hline \multicolumn{4}{|c|}{ All women } \\
\hline None & $660 / 529$ & $1.00^{\mathrm{b}}$ & \\
\hline$>0-<5$ & $225 / 366$ & $0.56(0.45-0.69)$ & $<0.001$ \\
\hline $5-<10$ & $28 / 42$ & $0.58(0.35-0.98)$ & 0.04 \\
\hline $10-<20$ & $12 / 13$ & $0.89(0.39-2.03)$ & 0.78 \\
\hline $20-<30$ & $45 / 43$ & $0.86(0.54-1.37)$ & 0.53 \\
\hline$\geqslant 30$ & $39 / 16$ & $2.33(1.26-4.31)$ & $<0.01$ \\
\hline \multicolumn{4}{|c|}{ Premenopausal women } \\
\hline None & $416 / 332$ & $1.00^{\mathrm{b}}$ & \\
\hline$>0-<5$ & $175 / 265$ & $0.62(0.48-0.79)$ & $<0.001$ \\
\hline $5-<10$ & $20 / 27$ & $0.62(0.33-1.17)$ & 0.14 \\
\hline $10-<20$ & $8 / 9$ & $0.85(0.31-2.34)$ & 0.75 \\
\hline $20-<30$ & $32 / 29$ & $0.86(0.49-1.50)$ & 0.59 \\
\hline$\geqslant 30$ & $21 / 9$ & $2.28(1.00-5.20)$ & 0.05 \\
\hline \multicolumn{4}{|c|}{ Postmenopausal women } \\
\hline None & $244 / 197$ & $1.00^{\mathrm{b}}$ & \\
\hline$>0-<5$ & $50 / 101$ & $0.41(0.27-0.62)$ & $<0.001$ \\
\hline $5-<10$ & $8 / 15$ & $0.49(0.19-1.24)$ & 0.13 \\
\hline $10-<20$ & $4 / 4$ & $0.97(0.23-4.06)$ & 0.96 \\
\hline $20-<30$ & $13 / 14$ & $0.97(0.42-2.26)$ & 0.95 \\
\hline$\geqslant 30$ & $18 / 7$ & $2.29(0.89-5.87)$ & 0.09 \\
\hline
\end{tabular}

Abbreviations: $\mathrm{Cl}=$ confidence interval; $\mathrm{OR}=$ odds ratio. ${ }^{\mathrm{a}}$ Estimates from unconditional logistic regression models included terms for age at interview (continuous), education (none, primary, secondary, tertiary), BMI (5-years ago), oral contraceptive use (never, ever), hormone replacement therapy (never, ever), breast cancer in first-degree relatives (no, yes), total energy intake (continuous), folate intake (continuous), tea drinking (no,

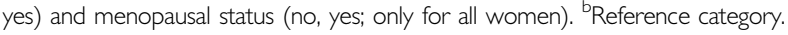

Table 4 Combined effect of dietary folate intake and alcohol consumption on breast cancer risk

\begin{tabular}{lllcl}
\hline $\begin{array}{l}\text { Ethanol intake } \\
(\mathbf{g} \text { per day) }\end{array}$ & $\begin{array}{l}\text { Folate intake } \\
(\boldsymbol{\mu} \text { g per day) }\end{array}$ & $\begin{array}{c}\text { No. Cases/ } \\
\text { controls }\end{array}$ & $\begin{array}{c}\text { OR } \\
(\mathbf{9 5} \% \mathbf{C l})^{\mathbf{a}}\end{array}$ & P-value $^{\mathbf{b}}$ \\
\hline Abstainers & Low & $245 / 177$ & $1.00^{\mathrm{b}}$ & \\
& Middle & $229 / 166$ & $0.70(0.51-0.95)$ & \\
& High & $186 / 186$ & $0.30(0.20-0.44)$ & \\
$>0-<15$ & Low & $70 / 136$ & $0.43(0.30-0.61)$ & $<0.001$ \\
& Middle & $99 / 154$ & $0.35(0.25-0.50)$ & \\
& High & $87 / 122$ & $0.23(0.15-0.36)$ & \\
& & & & \\
& Low 15 & $21 / 18$ & $0.93(0.47-1.84)$ & \\
& Middle & $21 / 12$ & $0.99(0.46-2.16)$ & \\
& High & $36 / 28$ & $0.38(0.20-0.72)$ & \\
& & &
\end{tabular}

Abbreviations: $\mathrm{Cl}=$ confidence interval; $\mathrm{OR}=$ odds ratio. ${ }^{\mathrm{a} E}$ stimates from unconditional logistic regression models included terms for age at interview (continuous) education (none, primary, secondary, tertiary), BMI (5-years ago), oral contraceptive use (never, ever), hormone replacement therapy (never, ever), breast cancer in first-degree relatives (no, yes), total energy intake (continuous), folate intake (continuous), tea drinking (no, yes) and menopausal status (no, yes; only for all women). ' Reference category.

$<15$ g per day for tumours with concordant but not discordant receptor status, that is, those with $\mathrm{ER}+/ \mathrm{PR}+$ and $\mathrm{ER}-/ \mathrm{PR}-$. The inverse association was more pronounced in postmenopausal women. Daily alcohol intake $\geqslant 15 \mathrm{~g}$ was significantly associated with tumours in postmenopausal women with discordant receptor status, that is, ER + /PR - or ER-/PR + (4.27, 1.57-11.65). There was no significant association with any joint hormone receptor subtype in premenopausal women.

\section{DISCUSSION}

Despite extensive investigation of the association between alcohol consumption and breast cancer risk, the conclusion remains controversial especially for low-to-moderate alcohol intake (Brown et al, 2010). Some studies reported that alcohol consumption was associated with a higher risk of incident breast cancer (SmithWarner et al, 1998; Bagnardi et al, 2001; Terry et al, 2006), while others based on more detailed consumption measures have found no relationship with breast cancer, particularly in premenopausal women (Garland et al, 1999; Kinney et al, 2000; Kropp et al, 2001; Baumgartner et al, 2002; Nagata et al, 2007; Bessaoud and Daurès, 2008; Brown et al, 2010; Kabat et al, 2010). This study was specifically designed to examine associations between low-tomoderate intake of alcohol and the risk of breast cancer by menopause status in the women and ER and PR status in the breast tumours. We observed that women who consumed alcohol at a low frequency and quantity had a reduced risk of breast cancer regardless of menopausal status. Compared with nondrinkers, those who consumed alcohol at $<5 \mathrm{~g}$ per day had a reduced breast cancer risk in pre- and postmenopausal women. For all tumour receptor subtypes combined, a small nonsignificant increased risk of higher alcohol intake ( $\geqslant 15 \mathrm{~g}$ daily) was generally associated with breast cancer in this study. There was no clear association between types of alcohol consumed and breast cancer risk, although significantly reduced risks from wine and beer drinking were observed in pre- and postmenopausal women, respectively.

The findings from this study that low-to-moderate alcohol intake was, if anything, inversely associated with risk of breast cancer are supported by results from other observational studies. One study recently reported that breast cancer risk was not significantly associated with alcohol drinking $(0.9,0.7-1.1)$ in Asian Americans including Chinese women, a population consuming alcohol at a low level with a median intake of $0.48 \mathrm{~g}$ per day in cases and $0.40 \mathrm{~g}$ per day in controls (Brown et al, 2010). Another 
Table 5 Association between alcohol intake (g per day) and breast cancer risk by hormone receptor status

\begin{tabular}{|c|c|c|c|c|c|c|c|c|c|}
\hline & \multirow[b]{2}{*}{$\begin{array}{c}\text { No. } \\
\text { controls }\end{array}$} & \multicolumn{2}{|r|}{ ER+ } & \multicolumn{2}{|r|}{ ER- } & \multicolumn{2}{|r|}{ PR+ } & \multicolumn{2}{|r|}{ PR- } \\
\hline & & $\begin{array}{l}\text { No. } \\
\text { cases }\end{array}$ & $\begin{array}{c}\text { OR } \\
(95 \% \mathrm{Cl})^{a}\end{array}$ & $\begin{array}{l}\text { No. } \\
\text { cases }\end{array}$ & $\begin{array}{c}\text { OR } \\
(95 \% \mathrm{Cl})^{\mathrm{a}}\end{array}$ & $\begin{array}{l}\text { No. } \\
\text { cases }\end{array}$ & $\begin{array}{c}\text { OR } \\
(95 \% \mathrm{Cl})^{\mathrm{a}}\end{array}$ & $\begin{array}{l}\text { No. } \\
\text { cases }\end{array}$ & $\begin{array}{c}\text { OR } \\
(95 \% \mathrm{Cl})^{a}\end{array}$ \\
\hline \multicolumn{10}{|l|}{ All women } \\
\hline None & 529 & 277 & $1.00^{\mathrm{b}}$ & 215 & $1.00^{\mathrm{b}}$ & 257 & $1.00^{\mathrm{b}}$ & 234 & $1.00^{\mathrm{b}}$ \\
\hline$>0-<15$ & 412 & 118 & $0.62(0.48-0.81)$ & 74 & $0.52(0.38-0.70)$ & 107 & $0.58(0.44-0.76)$ & 84 & $0.56(0.42-0.75)$ \\
\hline$\geqslant 15$ & 58 & 29 & $1.13(0.69-1.86)$ & 32 & $1.56(0.95-2.53)$ & 28 & $1.11(0.67-1.84)$ & 34 & $1.60(0.99-2.59)$ \\
\hline None & 332 & 184 & $1.00^{\mathrm{b}}$ & 128 & $1.00^{\mathrm{b}}$ & 178 & $1.00^{\mathrm{b}}$ & 133 & $1.00^{\mathrm{b}}$ \\
\hline$>0-<15$ & 297 & 89 & $0.64(0.47-0.87)$ & 54 & $0.57(0.40-0.83)$ & 86 & $0.63(0.46-0.86)$ & 56 & $0.59(0.4 \mid-0.85)$ \\
\hline$\geqslant 15$ & 38 & 19 & $1.03(0.56-1.92)$ & 19 & $1.47(0.78-2.76)$ & 22 & $1.21(0.67-2.18)$ & 17 & $1.35(0.70-2.61)$ \\
\hline \multicolumn{10}{|c|}{ Postmenopausal women } \\
\hline None & 197 & 93 & $1.00^{\mathrm{b}}$ & 87 & $1.00^{\mathrm{b}}$ & 79 & $1.00^{\mathrm{b}}$ & 101 & $1.00^{\mathrm{b}}$ \\
\hline$>0-<15$ & 115 & 29 & $0.56(0.34-0.94)$ & 20 & $0.37(0.21-0.68)$ & 21 & $0.46(0.26-0.8 I)$ & 28 & $0.47(0.28-0.79)$ \\
\hline
\end{tabular}

Abbreviations: $\mathrm{Cl}=$ confidence interval; $\mathrm{OR}=$ odds ratio. ${ }^{\mathrm{a} E s t i m a t e s ~ f r o m ~ u n c o n d i t i o n a l ~ l o g i s t i c ~ r e g r e s s i o n ~ m o d e l s ~ i n c l u d e d ~ t e r m s ~ f o r ~ a g e ~ a t ~ i n t e r v i e w ~(c o n t i n u o u s), ~ e d u c a t i o n ~}$ (none, primary, secondary, tertiary), BMI (5-years ago), oral contraceptive use (never, ever), hormone replacement therapy (never, ever), breast cancer in first-degree relatives (no, yes), total energy intake (continuous), folate intake (continuous), tea drinking (no, yes) and menopausal status (no, yes; only for all women). ${ }^{b}$ Reference category.

Table 6 Association between alcohol intake ( $g$ per day) and breast cancer risk by joint hormone receptor status

\begin{tabular}{|c|c|c|c|c|c|c|c|}
\hline & \multirow[b]{2}{*}{$\begin{array}{c}\text { No. } \\
\text { controls }\end{array}$} & \multicolumn{2}{|c|}{ ER+/PR+ } & \multicolumn{2}{|c|}{ ER-/PR- } & \multicolumn{2}{|c|}{$E R+/ P R-$ or $E R-/ P R+$} \\
\hline & & $\begin{array}{l}\text { No. } \\
\text { cases }\end{array}$ & $\begin{array}{c}\text { OR } \\
(95 \% \mathrm{Cl})^{a}\end{array}$ & $\begin{array}{l}\text { No. } \\
\text { cases }\end{array}$ & $\begin{array}{c}\text { OR } \\
(95 \% \mathrm{Cl})^{\mathrm{a}}\end{array}$ & $\begin{array}{l}\text { No. } \\
\text { cases }\end{array}$ & $\begin{array}{c}\text { OR } \\
(95 \% \mathrm{Cl})^{\mathrm{a}}\end{array}$ \\
\hline \multicolumn{8}{|l|}{ All women } \\
\hline None & 529 & 235 & $1.00^{\mathrm{b}}$ & 192 & $1.00^{\mathrm{b}}$ & 64 & $1.00^{\mathrm{b}}$ \\
\hline$>0-<15$ & 412 & 92 & $0.55(0.41-0.73)$ & 58 & $0.46(0.33-0.64)$ & 41 & $1.06(0.68-1.64)$ \\
\hline$\geqslant 15$ & 58 & 20 & $0.89(0.5 \mid-1.56)$ & 25 & $1.42(0.84-2.41)$ & 16 & $2.82(1.47-5.42)$ \\
\hline \multicolumn{8}{|c|}{ Premenopausal women } \\
\hline None & 332 & 160 & $1.00^{\mathrm{b}}$ & 109 & $1.00^{\mathrm{b}}$ & 42 & $1.00^{\mathrm{b}}$ \\
\hline$>0-<15$ & 297 & 72 & $0.58(0.42-0.8 I)$ & 39 & $0.49(0.32-0.74)$ & 31 & $1.08(0.64-\mid .81)$ \\
\hline$\geqslant 15$ & 38 & 16 & $0.99(0.5 \mathrm{I}-1.89)$ & 14 & $1.34(0.67-2.69)$ & 8 & $1.96(0.80-4.79)$ \\
\hline \multicolumn{8}{|c|}{ Postmenopausal women } \\
\hline None & 197 & 75 & $1.00^{\mathrm{b}}$ & 83 & $1.00^{\mathrm{b}}$ & 22 & $1.00^{\mathrm{b}}$ \\
\hline$>0-<15$ & 115 & 20 & $0.45(0.25-0.8 I)$ & 19 & $0.36(0.20-0.64)$ & 10 & $0.98(0.43-2.25)$ \\
\hline$\geqslant 15$ & 20 & 4 & $0.76(0.23-2.5 I)$ & 11 & $1.62(0.69-3.8 I)$ & 8 & $4.27(1.57-11.65)$ \\
\hline
\end{tabular}

Abbreviations: $\mathrm{Cl}=$ confidence interval; $\mathrm{OR}=$ odds ratio. ${ }^{\mathrm{a} E s t i m a t e s}$ from unconditional logistic regression models included terms for age at interview (continuous), residential area (urban, rural), education (none, primary, secondary, tertiary), BMI (5-years ago), age at menarche (continuous), oral contraceptive use (never, ever), hormone replacement therapy (never, ever), breast cancer in first-degree relatives (no, yes), total energy intake (continuous), folate intake (continuous), smoking (no, yes), tea drinking (no, yes), physical

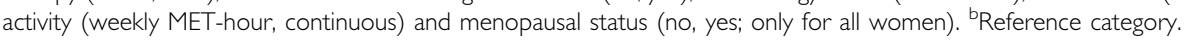

study conducted in France found that women who had an average consumption of $<1.5$ drinks per day had a lower risk $(0.58$, $0.34-0.97$ ) when compared with nondrinkers (Bessaoud and Daurès, 2008). Another study reported that alcohol intake of $<148$ g per week was associated with a reduced risk in nonHispanic Whites $(0.49,0.35-0.69)$, and that the protective effect was present in both pre- and postmenopausal women (Baumgartner et al, 2002). Furthermore, a reduced risk of breast cancer was found in premenopausal German women, whose average ethanol intake was $\leqslant 11 \mathrm{~g}$ per day defined for a lower-level consumption of alcohol (Kropp et al, 2001). A systematic review of three cohort studies and eight case-control studies concluded that there were inconsistent results regarding alcohol drinking and breast cancer risk in the Japanese population (Nagata et al, 2007).

Chinese women have presently a very low level of alcohol consumption. WHO reported that $61.2 \%$ of the population were lifetime abstainers and only $2.1 \%$ of them consumed three or more standard drinks on a typical drinking day although the WHO has noted that alcohol consumption in Asia is rising rapidly (WHO, 2004). In this study, $65.4 \%$ cases and $52.4 \%$ controls self-reported as lifetime abstainers, while the majority of current drinkers consumed $<10 \mathrm{~g}$ of alcohol per day, thus providing an opportunity to evaluate the association of low-to-moderate alcohol intake and breast cancer risk with reasonable precision. However, for the opposite reason, our study may be unsuitable to assess the effect of higher alcohol intake on breast cancer as few participants consumed at levels $\geqslant 30 \mathrm{~g}$ daily. Few other studies have investigated the association of breast cancer with alcohol consumption in Chinese women. One such study was performed in premenopausal Vietnamese and Chinese women and reported that those who consumed alcohol had an increased risk of breast cancer $(1.85,1.32-2.61)$ compared with nondrinkers but no details on alcohol intake available (Nichols et al, 2005). However, another study suggested that low alcohol intake was unrelated to breast cancer risk $(0.9,0.7-1.1)$ in Asian Americans including Chinese women (Brown et al, 2010).

It is unclear whether the relationship between alcohol consumption and breast cancer risk differs across ER and PR tumours subtype (Suzuki et al, 2008). In the study, we classified hormone receptor status separately and jointly and found that low-to-moderate alcohol intake was consistently associated with a reduced risk of all tumour receptor subtypes except where tumours had discordant receptor 
status, that is, $\mathrm{ER}+/ \mathrm{PR}-$ or $\mathrm{ER}-/ \mathrm{PR}+$. This risk reduction was evident in both pre- and postmenopausal women, but was more pronounced in postmenopausal women. There was no association between higher alcohol intake (daily $\geqslant 15 \mathrm{~g}$ ) and breast cancer risk with the exception of where discordant receptor status existed in postmenopausal women. There is no known biological basis for this result and we are reluctant to place much emphasis of it as an isolated finding in just one study.

Our findings regarding hormone receptor status are inconsistent to some extent with the results of other research. One study found that moderate alcohol consumption was associated positively with breast cancer in postmenopausal women with hormone receptor-positive tumours (Lew et al, 2009). A meta-analysis uncovered that an increase in alcohol consumption of $10 \mathrm{~g}$ per day was associated with statistically significant increased risks for all ER + , all ER-, ER $+\mathrm{PR}+$ and $\mathrm{ER}+\mathrm{PR}-$ but not ER-PR - tumours (Suzuki et al, 2008).

The biological mechanisms of the effects of alcohol on breast cancer aetiology have been widely discussed but remain to be characterised by detailed evidence. Investigators have suggested that alcohol consumption increases the risk of breast cancer in women by influencing oestrogen metabolism (Ginsburg, 1999). Alcohol appears to raise circulating oestrogen levels in premenopausal women and has a much more pronounced effect in postmenopausal women taking oestrogen replacements than in those not on replacement therapy (Ginsburg, 1999). The promotion of the growth of breast cancer may also be explained by an increased production by the liver of an insulin-like growth factor associated with moderate alcohol intake ( $\mathrm{Yu}$ et al, 2002). Moreover, alcohol may exhibit mutagenic and oxidative effects in breast tissue, affect folate levels and lead to damaged DNA (Dumitrescu and Shields, 2005). The investigation found that high intake of folate protect against the increased risk of breast cancer associated with high alcohol consumption in Chinese women, which supports the findings reported by previous studies (Baglietto et al, 2005; Beasley et al, 2010). Although several possible mechanisms link chronic alcohol consumption to breast cancer, it is possible that the critical effect of alcohol on breast cancer depends on a threshold level of exposure. Therefore, lack of adverse effects of low alcohol consumption on cancer found in our study and some others is plausible.

It is necessary to emphasis our study's limitations and strengths of our study when explain the finding. A strong feature was that detailed data were obtained on alcohol consumption habits as well as diet, lifestyle and factors relevant to hormonal status. A validated and reliable instrument with high intraclass correlations in our test-retest study (Zhang et al, 2005) and designed specifically for Chinese women was used to collect this information. Another feature of the study was the adjustment for caloric intake and other potential confounders such as hormone-related factors, which could affect the tumour hormone receptor status of breast cancers. The total energy intake of participants, which was derived from the FFQ, was comparable to other data on Zhejiang residents (Ge et al, 1995). Nevertheless, the case-control design may have also introduced certain sources of bias. The breast cancer cases were identified from medical records and interviewed in breast surgery wards, while the controls were randomly selected from outpatient clinics in the same hospitals by matching on the age of cases. A majority of patients are self-referred in China. Under the recruitment procedure, the cases and controls arose from the same populations of women using the hospitals and, thus, if a control was later to develop breast cancer it was highly likely that she would have been treated in the same hospital.

Selection bias was also minimised by selection procedures for controls that were unrelated to convenience or clinician contact. However, there was potential for selection bias from the choice of hospital controls. The type of hospital controls selected in the study could have differed from women who would have been treated in the hospital had they developed breast cancer. In our previous cancer case-control study, we recruited community controls in addition to hospital controls to evaluate differences in estimates of exposure distributions between two control groups. There was no difference in alcohol consumption, although some differences were found in the intake of salted vegetables and animal fat (Zhang et al, 2002). Another limitation of the study was that $25.2 \%$ cases had no ER/PR information. We assessed the effect of alcohol consumption on breast cancer risk using the cases without ER/PR information and the results obtained were similar to those using cases with ER/PR information.

Our research interest in alcohol consumption and breast cancer was apparent to the respondents at the time of the interview as detailed data were sought on consumption habits. However, in assessing the likelihood of any information bias, which we consider to have been low, it was relevant that no mention occurred in the popular media of any association between alcohol consumption and breast cancer before and at the time of the research. Although alcohol consumption, like other personal habits, can be reported by the subjects with reasonable accuracy, misclassification of exposure may still occur. However, such random errors are likely to bias results towards the null and would have been unlikely to account for the strongly inverse associations reported here. Exposure of cases to risk factors may change because of disease status. However, over $90 \%$ cases were newly diagnosed and interviewed within 3 months. It appears unlikely, therefore, that disease status materially affected the interview responses in reporting alcohol habits using a 'reference' recall period.

In conclusion, this study found that low alcohol intake $(<5 \mathrm{~g}$ daily) was inversely associated breast cancer in Chinese women. When considered according to hormone receptor status, the inverse association was consistently observed for all breast tumour receptor subtypes except those with a discordant receptor status, that is, $\mathrm{ER}+/ \mathrm{PR}-$ or $\mathrm{ER}-/ \mathrm{PR}+$. The reduced risk was evident in both preand postmenopausal women, but was more pronounced in postmenopausal women. An increased risk from daily alcohol intake of $\geqslant 15 \mathrm{~g}$ was found for tumours with a discordant receptor status in postmenopausal women. Given the number of women affected by breast cancer worldwide and the widespread consumption of alcohol, future studies are justified to fully understand the different effects of alcohol at low, moderate and high levels of intake on different breast tumour receptor subtypes according to hormone receptor status, including possible biological mechanisms.

\section{ACKNOWLEDGEMENTS}

We acknowledge with gratitude the participation of the Chinese women as subjects. We are grateful for the collaboration received from the participating hospitals and their staff. The first author is supported through a postdoctoral fellowship from the National Health and Medical Research Council (Australia, ID 303292).

\section{REFERENCES}

Ainsworth BE, Haskell WL, Whitt MC, Irwin ML, Swartz AM, Strath SJ, O’Brien WL, Bassett Jr DR, Schmitz KH, Emplaincourt PO, Jacobs Jr DR, Leon AS (2000) Compendium of physical activities: an update of activity codes and MET intensities. Med Sci Sports Exerc 32: S498-S504
Baglietto L, English DR, Gertig DM, Hopper JL, Giles GG (2005) Does dietary folate intake modify effect of alcohol consumption on breast cancer risk? Prospective cohort study. BMJ 331: 807

Bagnardi V, Blangiardo M, La Vecchia C, Corrao G (2001) A meta-analysis of alcohol drinking and cancer risk. Br J Cancer 85: 1700-1705 
Baumgartner KB, Annegers JF, McPherson RS, Frankowski RF, Gilliland FD, Samet JM (2002) Is alcohol intake associated with breast cancer in Hispanic women? The New Mexico Women's Health Study. Ethn Dis 12: $460-469$

Beasley JM, Coronado GD, Livaudais J, Angeles-Llerenas A, Ortega-Olvera C, Romieu I, Lazcano-Ponce E, Torres-Mejía G (2010) Alcohol and risk of breast cancer in Mexican women. Cancer Causes Control 21: 863-870

Bessaoud F, Daurès JP (2008) Patterns of alcohol (especially wine) consumption and breast cancer risk: a case-control study among a population in Southern France. Ann Epidemiol 18: 467-475

Brown LM, Gridley G, Wu AH, Falk RT, Hauptmann M, Kolonel LN, West DW, Nomura AM, Pike MC, Hoover RN, Ziegler RG (2010) Low level alcohol intake, cigarette smoking and risk of breast cancer in AsianAmerican women. Breast Cancer Res Treat 120: 203-210

Dumitrescu RG, Shields PG (2005) The etiology of alcohol-induced breast cancer. Alcohol 35: 213-225

Garland M, Hunter DJ, Colditz GA, Spiegelman DL, Manson JE, Stampfer MJ, Willett WC (1999) Alcohol consumption in relation to breast cancer risk in a cohort of United States women 25-42 years of age. Cancer Epidemiol Biomarkers Prev 8: 1017-1021

Ge KY, Zhai FY, Yan HC, Cheng L, Wang Q, Jia FM (1995) The dietary and nutritional status of Chinese populations in 1990s. ACTA Nutrimenta Sinica 17: $123-134$

Ginsburg ES (1999) Estrogen, alcohol and breast cancer risk. J Steroid Biochem Mol Biol 69: 299-306

Institute of Nutrition and Food Hygiene, (1999) Chinese Academic of Preventive Medicine. Food Composition Table (National Representative Values), 1st edn. People's Health Press: Beijing

Kabat GC, Kim M, Shikany JM, Rodgers AK, Wactawski-Wende J, Lane D, Powell L, Stefanick ML, Freiberg MS, Kazlauskaite R, Chlebowski RT, Wassertheil-Smoller S, Rohan TE (2010) Alcohol consumption and risk of ductal carcinoma in situ of the breast in a cohort of postmenopausal women. Cancer Epidemiol Biomarkers Prev 19: 2066-2072

Kinney AY, Millikan RC, Lin YH, Moorman PG, Newman B (2000) Alcohol consumption and breast cancer among black and white women in North Carolina (United States). Cancer Causes Control 11: 345-357

Kropp S, Becher H, Nieters A, Chang-Claude J (2001) Low-to-moderate alcohol consumption and breast cancer risk by age 50 years among women in Germany. Am J Epidemiol 154: 624-634

Lew JQ, Freedman ND, Leitzmann MF, Brinton LA, Hoover RN, Hollenbeck AR, Schatzkin A, Park Y (2009) Alcohol and risk of breast cancer by histologic type and hormone receptor status in postmenopausal women: the NIH-AARP Diet and Health Study. Am J Epidemiol 170: $308-317$

Longnecker MP (1994) Alcoholic beverage consumption in relation to risk of breast cancer: meta-analysis and review. Cancer Causes Control 5: 73-82
Nagata C, Mizoue T, Tanaka K, Tsuji I, Wakai K, Inoue M, Tsugane S (2007) Alcohol drinking and breast cancer risk: an evaluation based on a systematic review of epidemiologic evidence among the Japanese population. Jpn J Clin Oncol 37: 568-574

Nichols HB, Trentham-Dietz A, Love RR, Love RR, Hampton JM, Hoang Anh PT, Allred DC, Mohsin SK, Newcomb PA (2005) Differences in breast cancer risk factors by tumor marker subtypes among premenopausal Vietnamese and Chinese women. Cancer Epidemiol Biomarkers Prev 14: 41-47

Smith-Warner SA, Spiegelman D, Yaun SS, van den Brandt PA, Folsom AR, Goldbohm RA, Graham S, Holmberg L, Howe GR, Marshall JR, Miller AB, Potter JD, Speizer FE, Willett WC, Wolk A, Hunter DJ (1998) Alcohol and breast cancer in women: a pooled analysis of cohort studies. JAMA 279: $535-540$

Suzuki R, Orsini N, Mignone L, Wolk A (2008) Alcohol intake and risk of breast cancer defined by estrogen and progesterone receptor status-a meta-analysis of epidemiological studies. Int J Cancer 122: 1832-1841

Terry MB, Zhang FF, Kabat G, Britton JA, Teitelbaum SL, Neugut AI, Gammon MD (2006) Lifetime alcohol intake and breast cancer risk. Ann Epidemiol 16: $230-240$

USDA (2007) Table of Nutrient Retention Factors Release 6. Maryland: US Department of Agriculture, Agricultural Research Service,. Accessed on 8 June 2011. Available from URL: http://www.ars.usda.gov/Services/docs. htm?docid $=9448$

Whitemore AS, Wu-Willians AH, Lee M, Zheng S, Gallagher RP, Jiao DA, Zhou L, Wang XH, Chen K, Jung (1990) Diet, physical activity and colorectal cancer among Chinese in North American and China. J Natl Cancer Inst 82: $915-926$

WHO (2004) World Health Organization Global Status Report on Alcohol 2004. Country Profiles, Western Pacific Region-China. Accessed on 16 Jan 2011. Available from URL: http://www.who.int/substance_abuse/ publications/en/china.pdf

Yu H, Jin F, Shu XO, Li BD, Dai Q, Cheng JR, Berkel HJ, Zheng W (2002) Insulin-like growth factors and breast cancer risk in Chinese women. Cancer Epidemiol Biomarkers Prev 11: 705-712

Zhang M, Binns CW, Lee AH (2005) A quantitative food frequency questionnaire for women in southeast China: development and reproducibility. Asia Pac J Public Health 17: 29-34

Zhang M, Holman CDJ, Huang JP, Xie X (2007) Green tea and the prevention of breast cancer: a case-control study in Southeast China. Carcinogenesis 28: 1074-1078

Zhang M, Lee AH, Binns CW (2003) Physical activity and epithelial ovarian cancer risk: a case-control study in China. Int J Cancer 105: 838-843

Zhang M, Yang HJ, Holman CDJ (2009) Dietary intake of isoflavones and breast cancer risk by estrogen and progesterone receptor status. Breast Cancer Res Tr 118: 553-563

Zhang M, Yang ZY, Binns CW, Lee AH (2002) Diet and ovarian cancer risk: a case-control study in China. Br J Cancer 86: 712-717

This work is published under the standard license to publish agreement. After 12 months the work will become freely available and the license terms will switch to a Creative Commons Attribution-NonCommercial-Share Alike 3.0 Unported License. 